\title{
Immunofluorescent study of the expression of Ang1, Ang2 and Tie2 in the corpus cavernosum of the Rat during aging.
}

\author{
António Figueiredo***, Ana Lúcia Cordeiro****, Henrique Almeida*, Delminda Neves * \\ *Laboratory of Molecular Cell Biology of Faculty of Medicine and IBMC of Universidade do Porto \\ 4200-319 Porto, Portugal \\ **Both authors contributed equally to the presented work. \\ delmagal@med.up.pt
}

Aging is a recognized risk factor for erectile dysfunction (ED) contributing to vascular damage of penile tissue. Vascular system seems to be particularly prone to develop dysfunction along chronological aging, as supported by studies that associate aging with reduced endotheliumdependent dilation, atherosclerosis progression and angiogenic deficit in specific tissues. Cavernous tissue is particularly sensitive to atherosclerotic disease and to structural changes of the small vessels of the penis which conduces to functional impairment [1].

Vascular function in corpus cavernosum is progressively impaired with increasing age, in part, due to the decay in expression of VEGF (vascular endothelial growth factor) [2] that is an endothelial cell-specific mitogen, and is the major vascular growth factor involved in physiologic and pathologic angiogenesis. Nevertheless, other growth factors, such as angiopoietins, crosstalk in vivo with VEGF, modulating its effects. Both angiopoietin 1 (Ang1) and angiopoietin 2 (Ang2) depend on VEGF to regulate angiogenesis and both compete for binding to the endothelial-specific Tie2 receptor [3], in a context-dependent role. The crosstalk between angiopoietins and VEGF has an important modulating effect in vascular function. Thus, we proceeded to the study of vascular growth factors expression such as Ang1, Ang2 and their receptor Tie2 in corpus cavernosum of aged rat experimental model.

Male Wistar rats were divided in experimental groups $(n=6)$ according to age $(6,12,18$ and 24 months). Imunofluorescence detection of the growth factors was performed employing appropriate antibodies (Santa Cruz Biotechnology), using anti-Ang1 (1/25) or anti-Ang2 (1/25) combined with anti-Tie2 (1/200). Nuclei counterstaining was achieved with 4'-6-Diamino-2-phenylindole. Images were acquired in an Apotome microscope (Zeiss System, Göttingen, Germany). Semi-quantification was performed by Western blotting and labelled bands were evidenced using chemiluminescent substrate. Normalization of total Ang1, Ang2 and Tie2 was performed using beta-actin as an internal control.

We verified that Ang1 and Ang2 were localized mainly in smooth muscle at the periphery of vessel spaces, and also at endothelial cells. On the other hand, Tie 2 was strictly detected at the vascular endothelium. The calculated ratios of the expression of Ang1 and Ang2 in relation to the expression of their receptor Tie 2 decreases during aging, which suggest that blood vessel plasticity/remodeling can be compromised in the cavernous tissue of the aged rats.

\section{References}

[1] K.M. Azodzoi, BJU Int. 97 (2006) 11.

[2] D. Neves, J.Santos, N.Tomada, H. Almeida, P. Vendeira, Ann N Y Acad Sci 1067(2006) 164.

[3] L. Eklund, B. Olsen, Exp. Cell Res. 312 (2006) 630. 

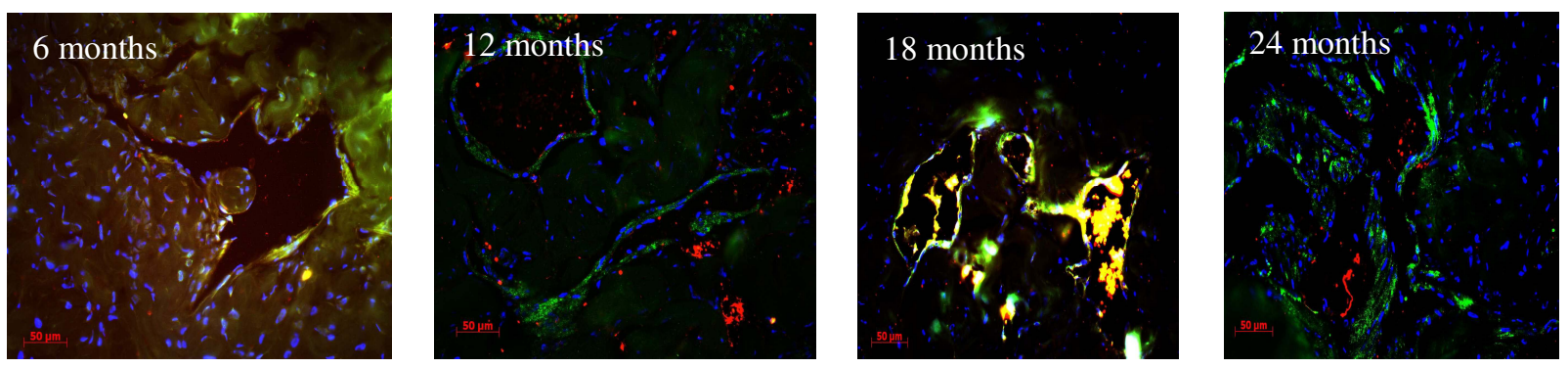

Fig. 1 - Ang1 (red) and Tie 2 (green) immunofluorescent detection on corpus caversosum of rats. Tie 2 was restricted to the endothelium and scarce expression of Ang 1 was observed both in endothelium and smooth muscle layer. Nuclei were stained in blue.
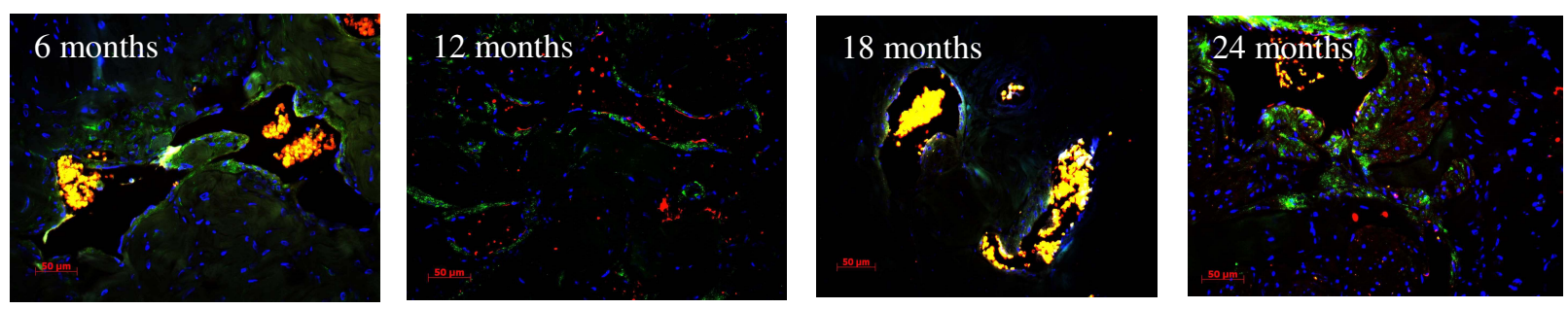

Fig. 2 - Ang 2 (red) and Tie 2 (green) immunofluorescent detection on corpus caversosum of rats. Tie 2 was restricted to the endothelium and Ang 2 presented similar localization to that observed for Ang1. Nuclei were stained in blue.
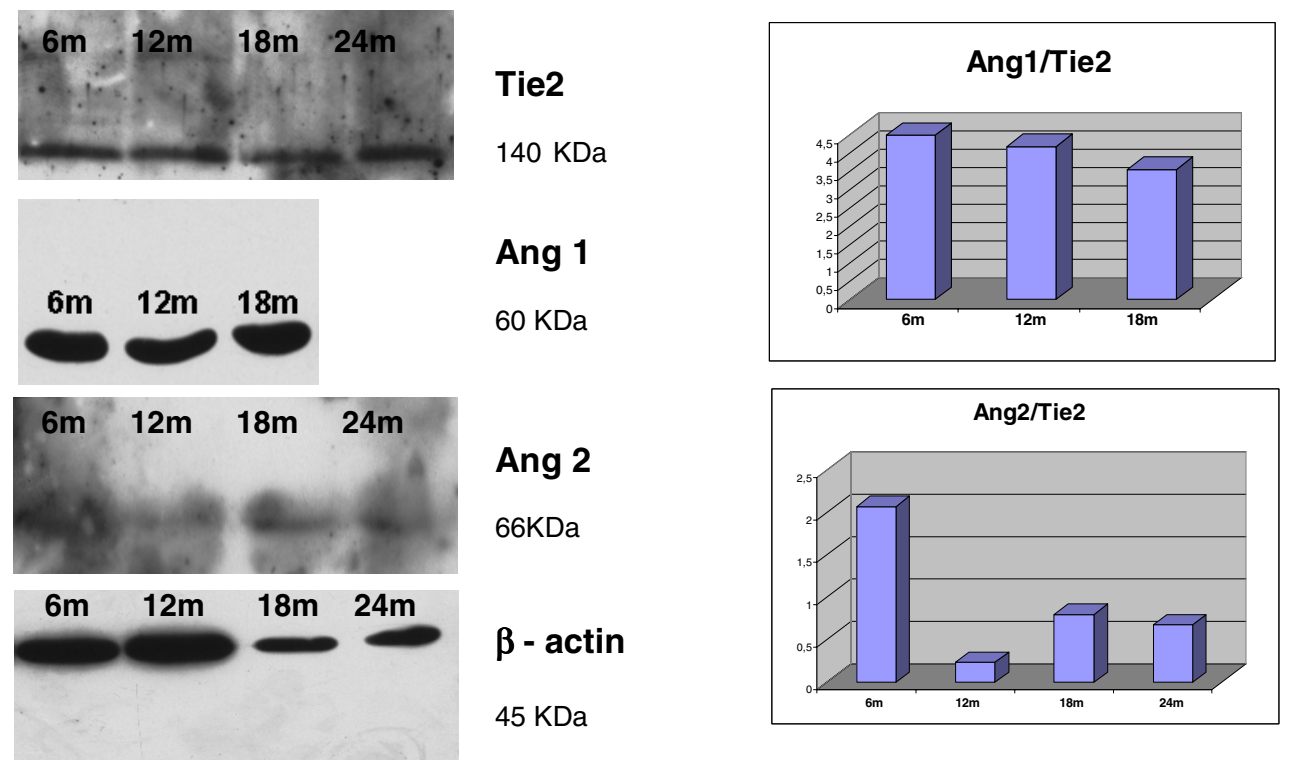

Fig. 3 - Western blotting semi-quantification of Tie2, Ang1 and Ang2 on the corpus caversosum of rats of 6, 12, 18 and 24 months of lifespan. The calculated ratios of Ang1/Tie2 and Ang2/Tie2 decrease along aging.

This study was supported by Caixa Geral de Depósitos/Universidade do Porto. 